\title{
Hacia la adopción de un tratado sobre empresas y derechos humanos: viejos debates, nuevas oportunidades*
}

\author{
Towards the adoption of a treaty on business \\ and human rights: old debates, new opportunities
}

\author{
Daniel Iglesias Márquez \\ Universidad de Sevilla \\ dimarquez@us.es
}

Sumario: Introducción. 1. Antecedentes en las Naciones Unidas: el camino hacia la regulación de las empresas en virtud del derecho internacional. 2. La Resolución $26 / 9$ y el proceso de elaboración del tratado sobre empresas y derechos humanos: apuntes desde la perspectiva Norte-Sur. 3. Hacia un tratado sobre empresas y derechos humanos: avances y retrocesos. 3.1. La prevención de abusos de derechos humanos cometidos en el marco de actividades empresariales. 3.2. El derecho de las víctimas de acceso a la justicia y a la reparación efectiva. 3.3. La asistencia y cooperación internacional para implementación del tratado. 3.4. Los mecanismos institucionales de supervisión y cumplimiento. Conclusiones. Bibliografía.

Resumen: El progresivo desarrollo de la agenda internacional sobre empresas y derechos humanos generó las condiciones necesarias para que, en junio de 2014, el Consejo de Derechos Humanos de las Naciones Unidas decidiera establecer un Grupo de Trabajo Intergubernamental de composición abierta para elaborar un instrumento jurídicamente vinculante, con el fin de regular las actividades de las empresas transnacionales y otras empresas en el derecho internacional de los derechos humanos. El rápido avance de las negociaciones entre los diferentes actores de interés permitió que en junio de

* Cómo citar: Iglesias Márquez, Daniel. 2019. "Hacia la adopción de un tratado sobre empresas y derechos humanos: viejos debates, nuevas oportunidades". Deusto Journal of Human Rights, 4: 145-176. http://dx.doi.org/18543/djhr-4-2019pp145-176 
2018 se presentara el Proyecto Cero del tratado sobre empresas y derechos humanos y, un año más tarde, una versión revisada del Proyecto. El presente artículo realiza un análisis crítico de los avances y retrocesos en el proceso de elaboración y de los elementos esenciales del futuro instrumento en la materia. Asimismo, reflexiona sobre el lugar que ocupa dicho instrumento en el derecho internacional contemporáneo.

Palabras clave: empresas, derechos humanos, Naciones Unidas, tratado, Principios Rectores, Norte-Sur.

Abstract: The progressive development of the international agenda on business and human rights generated the necessary conditions to establish an open-ended Intergovernmental Working Group by the United Nations Human Rights Council, whose task is the development of a legally binding instrument to regulate the activities of transnational corporations and other business enterprises in international human rights law. The progress of the round of negotiations between the different stakeholders allowed the publication of the Zero Draft of the treaty on business and human rights in July 2018 and the Revised Draft of the treaty in July 2019. This article makes a critical analysis of the advances and challenges of the treaty process and the key elements of the future instrument. It also reflects on the relevance of this instrument in contemporary international law.

Keywords: business, human rights, United Nations, treaty, Guiding Principles, North-South. 


\section{Introducción ${ }^{1}$}

Desde la década de los años setenta la agenda de las Naciones Unidas ha puesto en marcha diversos intentos y procesos - con diversos objetivos, alcances y estándares - para regular jurídicamente las actividades de las empresas en virtud del derecho internacional de los derechos humanos. Desde entonces ha emergido y evolucionado progresivamente la agenda internacional sobre empresas y derechos humanos, que incluye las acciones y esfuerzos de diversas organizaciones internacionales como la Organizaciones Internacional del Trabajo (OIT) y la Organización para la Cooperación y el Desarrollo Económicos (OCDE), entre otras. Esta agenda, con diversos matices y componentes multidisciplinarios, desarrolla un discurso que intenta alejarse de la filantropía de la responsabilidad social corporativa, para asegurar que las empresas respeten los derechos humanos en sus operaciones globales a través de estándares vinculantes. Los debates - aún inconclusos - en este ámbito, se enfocan en el estatus de las empresas y sus obligaciones en virtud del derecho internacional, así como en su responsabilidad jurídica en caso de abusos de derechos humanos causados en el desarrollo de sus actividades, entre otros.

Las diversas cuestiones señaladas - y otras- pretenden ser abordadas y resueltas mediante un futuro instrumento internacional vinculante sobre empresas y derechos humanos (Černič y Carrillo Santarelli 2018; Bilchitz 2016). Actualmente, en el seno del Consejo de Derechos Humanos (CDH) de las Naciones Unidas, está teniendo lugar un proceso de elaboración y adopción de un tratado en la materia que ha reavivado viejos y recurrentes debates sobre las obligaciones y responsabilidades de las empresas en el ámbito de los derechos humanos, pero en esta ocasión lo hace en un contexto de nuevas oportunidades y de mayor entendimiento del impacto de las empresas en los derechos humanos, que debería ayudar a que no se repitieran los anteriores intentos fallidos para alcanzar un instrumento de este tipo.

El proceso del tratado se ve respaldado por una movilización global sin precedentes por parte de la sociedad civil y varios Estados del Sur Global. Además, existe una inconformidad general sobre la eficiencia de las iniciativas voluntarias para regular el comportamiento de las empresas por sí solas. Este escenario ha acelerado el proceso del

1 El presente estudio ha sido realizado en el marco del proyecto de investigación «Gobernanza y Aplicación de la Responsabilidad Social Empresarial en la Unión Europea» (DER2017-85834-R). 
tratado y, en menos de cinco años, se ha publicado el Proyecto Cero del futuro instrumento, que dio inicio a la ronda de negociaciones sustantivas, y una versión revisada del Proyecto que integra los comentarios y sugerencias de los diversos actores que participan en el proceso de negociación.

El presente artículo responde a la pregunta de investigación sobre cuáles son las oportunidades y el potencial que representa un futuro instrumento vinculante en la materia, así como su complementariedad con otros instrumentos de soft law que comparten un objetivo común: asegurar el respeto de los derechos humanos en el contexto de las actividades empresariales. En primer lugar, se examina en qué medida otros procesos y desarrollos que han tenido lugar en el seno de las Naciones Unidas influyen en las negociaciones y delimitan el contenido del futuro instrumento. En segundo lugar, se reflexiona sobre el proceso de elaboración del tratado desde una perspectiva de NorteSur para entender los argumentos a favor y la razón fundamental de este instrumento, así como las motivaciones para que algunos Estados se opongan a su elaboración y adopción. En tercer lugar, se lleva a cabo un análisis crítico de los elementos esenciales que debe comprender un instrumento vinculante en la materia. Finalmente, se concluye con algunas reflexiones sobre el lugar que ocupa el futuro instrumento en el derecho internacional contemporáneo y su papel en la protección de los derechos humanos en la economía mundocapitalista.

\section{Antecedentes en las Naciones Unidas: el camino hacia la regulación de las empresas en virtud del derecho internacional}

El futuro tratado sobre empresas y derechos humanos, sin lugar a dudas, se ve influenciado y delimitado en gran medida por otros procesos similares y desarrollos alcanzados en el marco de las Naciones Unidas. La experiencia y contenido de estos precedentes no se puede obviar, sobre todo para poder superar las cuestiones en las que los diferentes actores implicados no se ponen de acuerdo y alcanzar la adopción y entrada en vigor del futuro instrumento que se discute hoy en día en esta materia.

El primer intento para regular internacionalmente a las empresas tuvo lugar en los debates de la Asamblea General de las Naciones Unidas sobre la soberanía permanente de los recursos naturales en de la década de los sesenta (GA Res 1803(XVII)). En 1974 se iniciaron 
las labores para la elaboración de un Código de Conducta para las Empresas Transnacionales (E/1988/39/Add.1). El Proyecto de Código estaba divido en dos secciones que pretendían alcanzar un equilibrio entre los derechos y las obligaciones de las empresas y de los Estados. La primera sección (Activities of Transnational Corporations) abordaba las actividades de las empresas transnacionales y contenía estándares de comportamiento directamente aplicables a las empresas. La segunda sección (Treatment of Transnational Corporations) se centraba en el trato general a las empresas transnacionales por parte de los países donde operaban. Finalmente, en un contexto de reajustes económicos y políticos en la comunidad internacional, el Código no fue adoptado oficialmente (Sauvant 2015).

En 2003, la Subcomisión de Promoción y Protección de los Derechos Humanos aprobó las Normas de las Naciones Unidas sobre Responsabilidades de las Empresas Transnacionales y otras Empresas Comerciales en la esfera de los Derechos Humanos (E/CN.4/ Sub.2/2003/12/Rev.2). Una de las particularidades de este instrumento fue que apostó por la adaptación de los estándares existentes en él, es decir, por una reformulación de los principios jurídicos internacionales para que fueran aplicables a las empresas.

Vázquez (2005), en relación con las Normas, señala que el documento pretendía ser una reafirmación de las obligaciones existentes para las empresas en virtud del derecho internacional de los derechos humanos. Sin embargo, sus autores reconocieron que en algunos aspectos las obligaciones establecidas en las Normas iban más allá del derecho internacional vigente. Las Normas intentaron imponer obligaciones vinculantes a las empresas transnacionales y otras empresas comerciales. El texto contemplaba que, aunque los Estados tienen la obligación primaria de proteger, respetar y garantizar los derechos humanos, las empresas también tenían obligaciones similares a las de los Estados (Weissbrodt y Kruger 2003).

Por ende, las Normas rompían la estructura clásica del derecho internacional y en ello residió la falta de apoyo político por parte de los Estados y de las empresas, que manifestaron fuertes reservas e hicieron hincapié en que un instrumento de este tipo no debía apartarse de la estructura tradicional del derecho internacional público, destacando así el papel central y fundamental de los Estados como sujetos únicos y exclusivos del derecho internacional (De Schutter 2016).

Tras el abandono de las Normas, el CDH estableció el mandato del profesor John Ruggie como Representante Especial del Secretario General para la cuestión de los Derechos Humanos y las Empresas Transnacionales y Otras Empresas. Ruggie concluyó su mandato en 
2011 con la aprobación unánime del CDH de los Principios Rectores de las Naciones Unidas sobre las Empresas y los Derechos Humanos (en adelante Principios Rectores) (A/HRC/17/31).

Los Principios Rectores rigen actualmente el ámbito y la agenda -nacional e internacional- en materia de empresas y derechos humanos (Deva y Bilchitz 2013). Este instrumento de soft law está basado en tres pilares, que agrupan 31 principios y sus comentarios respectivos: 1) la obligación de proteger los derechos humanos por parte de los Estados, 2) la responsabilidad de las empresas de respetar los derechos humanos y 3 ) el acceso a la reparación por parte de las víctimas de vulneraciones de derechos humanos.

Según Ruggie (2014), los Principios Rectores constituyen la primera plataforma de acción conjunta a nivel global para la creación de normativa común, así como para aclarar y asesorar políticamente a las empresas y a las administraciones sobre cómo proteger, respetar y cumplir con los derechos humanos, sin que ello obstaculice cualquier otro desarrollo normativo a largo plazo. De esta manera, otros instrumentos que contienen estándares internacionales de carácter no vinculante para las empresas, como las Líneas Directrices de la OCDE para Empresas Multinacionales o la Declaración Tripartita de la OIT de Principios sobre las Empresas Multinacionales y la Política Social, se han alineado con los Principios Rectores.

Los Principios Rectores se adecuan al statu quo del derecho internacional, caracterizado por una invisibilidad jurídica de las empresas que, como se señaló anteriormente, de acuerdo con la concepción clásica del derecho internacional, no son consideradas como titulares directas de obligaciones internacionales, y cuya virtualidad siempre depende de la mediación estatal (state-centric approach). La lógica operativa de los Principios Rectores consiste, por tanto, en que los Estados, sujetos obligados en virtud del derecho internacional, adopten las medidas necesarias para proteger los derechos humanos y garantizar el acceso a vías de reparación frente a posibles vulneraciones causadas por las actividades empresariales.

Está claro que el texto se aleja de la prescripción de obligaciones vinculantes para las empresas en virtud del derecho internacional y que, en su lugar, pone énfasis en el Estado como principal sujeto obligado a proteger los derechos humanos. Esta cuestión es una de las principales críticas a los Principios Rectores por parte de la academia y las organizaciones de la sociedad civil. No obstante, el state-centric approach de los Principios Rectores ha sido clave para alcanzar un conceso político entre los diversos grupos de interés en la materia. 
A pesar de las críticas y el lento proceso de implementación de los Principios Rectores a nivel nacional, este instrumento marcó un punto de inflexión en la agenda de las Naciones Unidas sobre el tema de empresas y derechos humanos. Como indica Ruggie (2014), la adopción de los Principios Rectores se trató de la fase final del comienzo de un proyecto que necesita continuar construyendo un andamiaje jurídico a nivel internacional para garantizar la protección y realización de los derechos humanos en el contexto de las actividades empresariales. Si bien el Proyecto de Código y las Normas son referentes importantes a tener en cuenta durante el proceso de elaboración y negociación del futuro tratado sobre empresas y derechos humanos, sobre todo para evitar que las discrepancias del pasado afecten la oportunidad histórica de adoptar el primer instrumento internacional de carácter vinculante en la materia, los Principios Rectores, por su parte, orientan y sientan las bases para configurar el proyecto de tratado.

De hecho, como se analiza en los siguientes epígrafes, el tratado y los Principios Rectores no son contradictorios, sino más bien se complementan mutuamente para avanzar en los desarrollos normativos y prácticos que favorezcan la protección y reparación de las víctimas de abusos de derechos humanos cometidos en el marco de las actividades empresariales, especialmente en los Estados incapaces o no dispuestos a cumplir con sus obligaciones internacionales frente a las empresas. En este sentido, el tratado es concebido como una repuesta para abordar las limitaciones y superar los obstáculos que presentan los Principios Rectores, sobre todo en relación con la implementación a nivel nacional del tercer pilar sobre el acceso a los mecanismos de reparación, que ha recibido poca atención por parte de los Estados.

En suma, el tratado sobre empresas y derechos humanos no es ajeno a la agenda de las Naciones Unidas, sino más bien se trata de una cuestión pendiente en los últimos cincuenta años. El proceso de elaboración y negociación de dicho instrumento no solo se nutre de los Principios Rectores, sino de la experiencia de otros procesos similares en el seno de las Naciones Unidas, que marcan importantes pautas y los límites diplomáticos para la posible adopción del tratado. Los Principios Rectores y la iniciativa internacional del tratado persiguen un objetivo común que consiste en aportar soluciones a problemas de acción colectiva, como son los impactos de las operaciones empresariales globales en los derechos humanos. 


\section{La Resolución $\mathbf{2 6 / 9}$ y el proceso de elaboración del tratado sobre empresas y derechos humanos: apuntes desde la perspectiva Norte-Sur}

El CDH adoptó en julio 2014 la Resolución 26/9 relativa a la «Elaboración de un instrumento internacional jurídicamente vinculante sobre las empresas transnacionales y otras empresas comerciales en materia de derechos humanos»(A/HRC/RES/26/9), a cargo de un Grupo de Trabajo Intergubernamental (GTI) de composición abierta. Esta resolución condujo al histórico proceso de elaboración de un instrumento de hard law en el ámbito de empresas y derechos humanos. Asimismo, polarizó las opiniones entre los actores de interés y abrió, una vez más, el debate recurrente en la materia sobre las normas vinculantes y no vinculantes para regular directamente las actividades de las empresas en virtud del derecho internacional. Esta división responde principalmente a las posiciones encontradas entre los que apoyan la implementación de los Principios Rectores, alineados con el statu quo del derecho internacional, y los que consideran importante y oportuno la adopción de un tratado en el ámbito de empresas y derechos humanos que ponga a prueba las fronteras del derecho internacional.

La Resolución 26/9 responde principalmente a las demandas de los Estados del Sur Global -y de la sociedad civil- de cubrir los vacíos existentes en relación con la gobernanza global de las actividades de las empresas y, sobre todo, para nivelar la asimetría normativa en favor de las empresas que gozan de derechos sustantivos y de reparación, pero carecen de obligaciones directas en el marco del derecho internacional (Hernández Zubizarreta 2016).

En este sentido, cabe destacar que el Estado de Ecuador comenzó a promover durante la vigésimo cuarta sesión del CDH la necesidad de contar con un instrumento internacional para regular las actividades empresariales ante los impactos negativos en los derechos humanos (República del Ecuador 2013). Este país, como muchos otros del Sur Global, ha experimentado las dificultades y debilidades que presentan los ordenamientos jurídicos, tanto a nivel nacional como internacional, para prevenir y hacer efectiva la responsabilidad de las empresas por los impactos negativos causados en el desarrollo de sus actividades. Por ejemplo, en el caso Chevron-Texaco en Ecuador, la Corte Provincial de Sucumbíos dictó en 2011 una sentencia a favor de las personas afectadas por los impactos ambientales causados por las actividades de la empresa, reconociendo el derecho a una compensación por los daños sufridos. Sin embargo, la empresa carece de patrimonio 
ejecutable en Ecuador, lo que ha llevado a que las personas afectadas busquen el reconocimiento y la ejecución de la sentencia en otras jurisdicciones, como Canadá, Brasil y Argentina, sin éxito hasta el momento (León y Liu 2018).

Sudáfrica respondió de manera positiva a la proposición y, junto con Ecuador, presentaron la propuesta de resolución que sería apoyada por las organizaciones de la sociedad civil y por algunos Estados del Sur Global, como Argelia, Benín, Burkina Faso, China, Congo, Costa de Marfil, Cuba, Etiopía, Rusia, Filipinas, India, Indonesia, Kazajstán, Kenia, Marruecos, Namibia, Pakistán, Venezuela y Vietnam, que consideran insuficientes los Principios Rectores para generar un cambio de paradigma en el modus operandi de las empresas. Mientras tanto, los Estados del Norte Global, donde tienen su sede diversas empresas transnacionales, y las economías emergentes con un modelo de mercado neoliberal votaron en contra o se abstuvieron.

La dividida votación de la Resolución $26 / 9$ no solo constata la dicotomía Norte-Sur, que se acentúa en la economía mundo-capitalista, sino también es un reflejo del contexto geopolítico y económico en el que operan las empresas. Es decir, la mayoría de host States del Sur Global se ven en muchas ocasiones sobrepasados por el poder de las grandes empresas y consideran necesario crear un instrumento internacional que logre un equilibrio de poderes. Por su parte, los home States del Norte Global y de las economías emergentes que se benefician de la internacionalización de sus empresas ven peligrar el estatus privilegiado del que gozan sus empresas transnacionales $\mathrm{y}$, por consiguiente, se oponen a las negociaciones de un instrumento internacional que sea jurídicamente vinculante para las empresas o altere las dinámicas de la economía global.

Según el mandato de la Resolución 26/9, el GTI, presidido desde su creación por Ecuador, se reuniría en varios períodos de sesiones para elaborar y deliberar constructivamente el contenido, el alcance, la naturaleza y la forma del futuro tratado. Los dos primeros períodos de sesiones del GTI recogieron, por tanto, dichas deliberaciones constructivas sobre el futuro instrumento internacional. En el tercer período de sesiones se debatió sobre el «Documento de Elementos para el proyecto de instrumento internacional jurídicamente vinculante sobre empresas transnacionales y otras empresas con respecto a los Derechos Humanos». Este Documento, presentado por el PresidenteRelator del GTI, constituyó un paso clave en la redacción del Proyecto Cero del tratado sobre empresas y derechos humanos, ya que refleja los insumos y los debates realizados por los Estados y otras partes relevantes en el marco de los primeros períodos de sesiones. 
La Resolución 26/9 no especifica el mandato del GTI después de las tres primeras sesiones. Sin embargo, el informe adoptado en la tercera sesión comprometió al Presidente-Relator a presentar un borrador de texto articulado para la siguiente sesión (CDH 2018). Así, se aseguró un cuarto período de sesiones, que tuvo lugar en 2018 y en el que se llevaron a cabo las primeras negociaciones sustantivas sobre el Proyecto Cero del tratado sobre empresas y derechos humanos, que se analiza en los siguientes apartados. Los subsecuentes períodos de sesiones son, por tanto, estratégicos y se plantean llenos de obstáculos políticos para seguir avanzando en la construcción de un instrumento vinculante sobre empresas y derechos humanos a nivel internacional.

Los debates en los períodos de sesiones del GTI han tenido lugar en un ambiente de confrontación política e ideológica entre los Estados del Sur Global, apoyados por las organizaciones de la sociedad civil, y los Estados del Norte Global, respaldados por las empresas (Cassel 2018b). Es decir, mientras que la gran mayoría de los Estados del Sur Global se muestran más abiertos a la idea de un tratado que ponga a prueba los límites del derecho internacional, los Estados del Norte Global son más reticentes a la adopción de un instrumento que pueda afectar los consensos globales alcanzados en el ámbito de empresas y derechos humanos.

Los Estados miembros de la Unión Europea (UE), Estados Unidos, Japón y Corea del Sur son algunos de los países que se oponen a la adopción del tratado. Según estos Estados, el tratado amenaza la implementación de los Principios Rectores y aún existen dudas sobre la aplicación práctica de un instrumento internacional de hard law para regular las actividades de las empresas. Esta ha sido la posición que la UE ha mantenido desde el inicio de los períodos de sesiones, argumentando que el proceso del tratado es incompatible con los Principios Rectores. La participación de la UE y de sus aliados comerciales en las negociaciones parece intentar dilatar y obstaculizar el mandato del GTI. Los Estados Unidos, por su parte, no solo se oponen al tratado argumentando la falta de tiempo suficiente para implementar los Principios Rectores, sino que rechazaron participar en los períodos de sesiones del GTI (Delegación de los Estados Unidos 2014). Este contexto de opiniones encontradas afecta a las deliberaciones constructivas y genera dificultades para encontrar un balance entre los intereses de los diversos actores que participan en las negociaciones del tratado.

Con una visión más innovadora del derecho internacional, algunos Estados del Sur Global, junto con las organizaciones de la sociedad civil, proponen que el tratado debe contener obligaciones directas de 
carácter internacional para las empresas. En este sentido, el Estado de Ecuador señala que el futuro instrumento vinculante debe incluir obligaciones para las empresas, tales como la diligencia debida hacia sus empleados y hacia las comunidades en las que operan (Delegación de Ecuador 2017). En un sentido similar, la Delegación de Bolivia considera que el tratado debe articular obligaciones, claras y directas para las empresas transnacionales, para que puedan ser consideradas responsables de prevenir, mitigar y compensar por los efectos de sus actividades en los derechos humanos, a fin de evitar la impunidad por sus acciones (Delegación de Bolivia 2017).

En el derecho internacional no existe ningún obstáculo para configurar obligaciones jurídicas de carácter vinculante para las empresas, cuyo cumplimiento podría hacerse efectivo a través de organismos internacionales competentes (Iglesias 2018b). Sin embargo, esta es la cuestión que mayor oposición política ha encontrado por parte de los Estados del Norte y las empresas. La UE sostiene que los agentes sujetos al ámbito de aplicación del futuro instrumento deben ser los Estados y, por ello, ha cuestionado al GTI sobre cuál es el fundamento jurídico para imponer obligaciones internacionales de derechos humanos a las empresas (Delegación de la Unión Europea 2017; A/HRC/37/67). En esta misma línea, las organizaciones internacionales del sector privado defienden la postura de que cualquier nuevo tratado debe evitar imponer obligaciones directas a las empresas que sean aisladas de las de los Estados (IOE et al. 2016).

Otra de las cuestiones que ha generado tensiones entre el Sur y el Norte Global es el establecimiento de una jerarquía de las normas en el derecho internacional. Es decir, que el tratado establezca de forma explícita la supremacía de los derechos humanos sobre los instrumentos internacionales en materia de inversión (CDH 2016). Las "cláusulas de estabilización» contempladas en los tratados de inversión afectan principalmente los poderes soberanos de los Estados del Sur Global. Sobre todo restringen la capacidad normativa y regulatoria para garantizar de manera efectiva el respeto de los derechos humanos y la protección del medio ambiente (Miles 2010). Estas cláusulas previenen que los Estados receptores de inversiones realicen eventuales reformas legislativas o regulatorias. De lo contrario, están sujetos al riesgo de ser demandados ante tribunales arbitrales internacionales, constituidos para la solución de controversias entre Estados e inversores extranjeros por supuestas políticas discriminatorias o por ajustes en los contratos.

Diversos casos ante estas instancias internacionales de solución de controversias demuestran que los laudos arbitrales tienden a proteger los intereses de las empresas cuando consideran que los derechos 
relativos a su inversión han sido vulnerados por la acción regulatoria del Estado (Brown 2013). Estos casos resultan particularmente costosos para los Estados del Sur Global debido a que tienen que pagar considerables indemnizaciones a las empresas, sin mencionar el pago de los costes judiciales. Cabe resaltar que la jurisprudencia de los tribunales arbitrales rara vez hace referencia a los instrumentos de derechos humanos, ya que están fuera del ámbito de competencia ratione materiae del tribunal. En consecuencia, los tribunales arbitrales suelen priorizar en la práctica el marco jurídico previamente acordado por las partes antes que los tratados internacionales en materia de derechos humanos o medio ambiente.

El anteriormente señalado caso Chevron-Texaco en Ecuador ha dado lugar a un complejo y polémico procedimiento arbitral al amparo del Tratado Bilateral de Inversiones firmado entre Ecuador y Estados Unidos. El laudo dictado por la Corte Permanente de Arbitraje (CPA) en 2019 no solo anula la sentencia dictada en 2011 a favor de los afectados por las actividades de la empresa, sino también impone al Estado la obligación de pagar una indemnización por los daños y perjuicios causados a la empresa por la sentencia. ${ }^{2}$ La CPA determinó que el Estado falló en garantizar la justicia a la empresa y violó sus derechos procedimentales, incluido el derecho a la defensa. Por tanto, este laudo legitima los impactos negativos causados por las actividades de las empresas en Ecuador. Asimismo, perpetúa el contexto de impunidad en el que opera la empresa y, sobre todo, menoscaba la soberanía del Estado en beneficio del poder corporativo.

Finalmente, las obligaciones extraterritoriales también generan desacuerdo entre los Estados del Sur y Norte Global. El derecho internacional contemporáneo no prohíbe el ejercicio de la jurisdicción extraterritorial de los Estados para regular y hacer efectiva la responsabilidad de las empresas domiciliadas o registradas en su territorio o bajo su control, siempre que haya una base jurisdiccional reconocida. Algunas delegaciones y organizaciones de la sociedad civil consideran que el tratado debe incluir normas claras para determinar que los Estados de origen tienen deberes en relación con las operaciones extraterritoriales de sus empresas y que esos deberes no menoscaban la soberanía de terceros Estados (Cassel 2018a).

En este sentido, diversos órganos creados en virtud de los tratados de derechos humanos han recomendado a algunos Estados del

2 Véase Chevron Corporation and Texaco Petroleum Corporation v. The Republic of Ecuador, UNCITRAL, PCA Case No. 2009-23. 
Norte Global la adopción de medidas para impedir que las empresas registradas en su jurisdicción cometan abusos en el extranjero. El Comité de Derechos Económicos, Sociales y Culturales, por ejemplo, recomendó al Reino Unido la adopción de medidas legislativas y administrativas apropiadas para asegurar la responsabilidad legal de las empresas domiciliadas en su jurisdicción respecto a las violaciones de los derechos económicos, sociales y culturales en sus proyectos en el extranjero, cometidas directamente por esas empresas o resultantes de las actividades de sus filiales (E/C.12/GBR/CO/6). Por su parte, el Comité de Derechos Humanos ha alentado a Canadá y a Alemania a que establezcan claramente que todas las empresas domiciliadas en su territorio o bajo su jurisdicción deben respetar en todas sus actividades (incluidas las que se realizan en el extranjero) la normativa de los derechos humanos de conformidad con el Pacto Internacional de Derechos Civiles y Políticos (CCPR/C/CAN/CO/6; CCPR/C/DEU/CO/6).

No obstante, algunos Estados son reticentes al ejercicio de la jurisdicción extraterritorial para la prevención y reparación de violaciones de derechos humanos cometidas por sus empresas en el extranjero. Esto es debido a que se asume que esta práctica puede crear distorsiones y desventajas competitivas en el mercado global en relación con otras empresas locales o extranjeras, cuyos Estados de origen no les exigen los mismos estándares.

Las propuestas y narrativas de los Estados del Sur Global son insumos importantes en el proceso de elaboración y negociación del tratado. Desde los tiempos de las colonias hasta el día de hoy, el derecho internacional, influenciado por la ideología y los valores del Norte Global, ha favorecido la acumulación y la expansión de las actividades de las empresas (Anghie 2006). Incluso la doctrina ha llegado a considerar que el derecho internacional de los derechos humanos ha sido utilizado por el Norte Global como una herramienta al servicio de la globalización para ocultar y perpetuar la dominación y subordinación —política y económica - del Sur Global (González 2015). El tratado sobre empresas y derechos humanos, por tanto, es un instrumento con potencial para transformar el contexto global y puede aspirar a constituir un orden internacional alternativo más equitativo y justo, si se toman en consideración el discurso y las perspectivas del Sur Global. Este instrumento puede generar un contrapeso a la hegemonía de los modelos de consumo y de desarrollo económico del Norte Global basados en la acumulación de bienes, el control de la naturaleza, el crecimiento económico ilimitado y el rechazo del conocimiento, las prácticas y las creencias indígenas por ser considerados obstáculos para la «modernización»(Gordon y Sylvester 2011). Asimismo, permitiría 
alzar las voces de las víctimas de abusos corporativos en el Sur Global. En este sentido, Simons (2012) sugiere que un tratado en la materia es clave para cambiar el equilibrio de poder entre, por un lado, los actores corporativos transnacionales y, por otro lado, los Estados anfitriones del Sur Global y las víctimas de abusos corporativos de derechos humanos.

\section{Hacia un tratado sobre empresas y derechos humanos: avances y retrocesos}

La publicación del Proyecto Cero en julio de 2018 fue uno de los pasos más importantes en el complejo proceso de elaboración del tratado sobre empresas y derechos humanos que ha tenido lugar en los últimos años en el seno del CDH. Se trató de una oportunidad histórica para que los Estados llevaran a cabo por primera vez negociaciones sustantivas y constructivas sobre un instrumento internacional vinculante en la materia. Junto con el Proyecto Cero, en septiembre de 2018, el GTI publicó un Proyecto de Protocolo Facultativo.

El Proyecto Cero de 2018 generó diversas opiniones encontradas entre la sociedad civil, organizaciones internacionales, academia y organizaciones empresariales, ya que muchas de las disposiciones propuestas en esta versión eran imprecisas, incoherentes e inconsistentes en relación con otras del mismo texto e incluso algunas eran reiterativas con obligaciones y principios generales de derecho internacional.

En julio de 2019 se presentó una versión revisada del Proyecto que no solo presenta mejoras cualitativas y sustanciales, sino también una estructura, contenido y lenguaje mucho más preciso, coherente y claro (Cassel 2019). ${ }^{3}$ A su vez, es un documento que intenta alcanzar un mayor consenso diplomático y conciliar las posiciones entre las partes a favor del tratado y las que se posicionan en contra de un instrumento vinculante que pueda afectar la implementación de los Principios Rectores. El Preámbulo de la versión revisada de 2019 no solo utiliza un lenguaje más próximo a los Principios Rectores, sino también reconoce el papel que han jugado en la implementación del Marco de las Naciones Unidas de «Proteger, Respetar y Remediar». Esta referencia a los Principios Rectores puede ser considerada como un llamado a los detractores del tratado a participar con un criterio constructivo y trabajar con un espíritu de colaboración durante las negociaciones,

3 La versión revisada del Proyecto será debatida en el quinto período de sesiones, que tendrá lugar del 14 al 18 de octubre de 2019 en el CDH (Ginebra). 
teniendo en cuenta la compatibilidad y complementariedad entre ambos instrumentos.

El régimen internacional propuesto hasta la fecha en materia de empresas y derechos humanos contiene un enfoque orientado a garantizar el acceso a la justicia a las víctimas de abusos corporativos. Uno de los objetivos propuestos para el futuro instrumento es asegurar el acceso efectivo a la justicia y la reparación a las víctimas de violaciones y abusos de derechos humanos cometidos por empresas (artículo 2 del Proyecto Cero y de la versión revisada del Proyecto). Por tanto, se trata de empoderar a las víctimas a través de un tratado cuyas disposiciones están principalmente dirigidas a cubrir los vacíos jurídicos existentes en el acceso a los mecanismos de reparación - a nivel internacional y nacional- en caso de abusos corporativos de derechos humanos, así como para hacer efectiva la responsabilidad jurídica de las empresas implicadas. Por ello, uno de los posibles valores añadidos del futuro tratado, junto con su protocolo facultativo, a la agenda internacional sobre empresas y derechos humanos es que contiene disposiciones que implementan el tercer pilar de los Principios Rectores sobre el acceso a los mecanismos de reparación.

Otra de las novedades evidentes de la versión revisada de 2019 es la ampliación del ámbito de aplicación del futuro instrumento a través de un enfoque híbrido («hybrid approach») (Deva 2018a). Las disposiciones sustantivas del Proyecto Cero estaban previstas para ser aplicadas a las violaciones de los derechos humanos ocurridas en el contexto de toda "actividad empresarial de carácter transnacional». De esta manera, se intentaba diluir la controversia que ha surgido sobre si el tratado debe enfocarse únicamente a la actuación de las empresas y otras entidades cuya actividad es de carácter transnacional, sin aplicarse a las empresas locales registradas con arreglo a la legislación nacional (Deva 2018b). En la versión revisada del Proyecto se propone que el instrumento se aplicará, salvo que se indique lo contrario, a «todas las actividades empresariales», incluidas particularmente, pero no limitadas, a aquellas de carácter transnacional. De esta manera no solo se reafirma la obligación de los Estados de controlar y regular a las empresas dentro de su territorio o bajo su jurisdicción, sino también se crean obligaciones respecto a las actividades transnacionales de las empresas. A su vez, el nuevo ámbito de aplicación propuesto parece ser más coherente con la propuesta de la UE de no limitar la discusión exclusivamente a las empresas transnacionales. Con este nuevo enfoque se reducen los argumentos de la UE $-\mathrm{y}$ otros Estados y actores con posturas similares- para no contribuir a alcanzar el mandato del GTI. 
El Proyecto Cero y la versión revisada del Proyecto de 2019 indican que el futuro instrumento se alejará de posturas que alterarían el statu quo del derecho internacional. Es decir, no se contemplan obligaciones directas para las empresas de respetar los derechos humanos, sino más bien se reafirma la obligación primaria de los Estados de, por un lado, proteger y respetar los derechos humanos frente a las actividades empresariales y, por otro lado, garantizar a las víctimas recursos efectivos en caso de violaciones. Tampoco se ha logrado incluir ninguna referencia expresa sobre la primacía de los instrumentos de derechos humanos que pudieran corregir el desequilibrio de facto en el régimen internacional respecto a los acuerdos de comercio e inversión.

Como se observa más adelante, la cuestión en la que quizás se avanza más - de manera cautelosa - es el ejercicio de la jurisdicción adjudicativa de carácter extraterritorial. Si bien el lenguaje utilizado en el Proyecto Cero y en la versión revisada no hace referencia a obligaciones extraterritoriales, varias de las disposiciones propuestas tienen implicaciones más allá de las fronteras de un Estado. Por tanto, contribuyen a reafirmar que en la economía mundo-capitalista las obligaciones de los Estados en relación con la protección de los derechos humanos no terminan en sus fronteras territoriales.

Debido a la extensión limitada del presente artículo, resulta imposible llevar a cabo un análisis crítico de todas las disposiciones que deberían estar incluidas en el tratado sobre empresas y derechos humanos. Por ello, se han elegido cuatro elementos que se consideran esenciales en el futuro instrumento para generar un cambio en el paradigma global de «business as usual».

\subsection{La prevención de abusos de derechos humanos cometidos en el marco de actividades empresariales}

Los diversos actores que participan en los períodos de sesiones del GTI coinciden en que la prevención es un elemento primordial del futuro instrumento con el fin de evadir los costos de complejos litigios y, sobre todo, para evitar el sufrimiento de las víctimas (CDH 2019; Cantú 2018; Guamán 2018). El artículo 9 del Proyecto Cero del tratado disponía que los Estados Parte velarán porque en su legislación interna todas las personas con actividades empresariales de carácter transnacional en su territorio o bajo su jurisdicción o control respeten las obligaciones de diligencia debida. 
Esta disposición de prevención del Proyecto Cero recogía y reinterpretaba algunos de los elementos de la diligencia debida en materia de derechos humanos contemplada en los Principios Rectores y desarrollada en las guías sectoriales de diligencia debida de la OCDE. ${ }^{4}$ De acuerdo con los Principios Rectores, los pasos clave del proceso de diligencia debida son: i) evaluar las consecuencias negativas reales o potenciales sobre los derechos humanos; ii) integrar las conclusiones de sus evaluaciones de impacto en sus funciones y procesos internos; iii) dar un seguimiento de la eficacia de las medidas adoptadas; y, iv) comunicar al exterior sobre cómo se abordan los impactos.

El artículo 9 del Proyecto Cero incluyó en gran medida estos pasos en sus disposiciones. Sin embargo, también omitió algunos como la integración de los resultados de las evaluaciones en las operaciones de las empresas y la comunicación con terceros interesados sobre cómo se abordan los impactos (Fasciglione 2018). En este sentido, Guamán (2018) identifica, en el Proyecto Cero, la falta de la obligación de efectuar una política de publicidad vinculada directamente con los resultados de los mecanismos de supervisión, identificación y evaluación.

Como aspecto positivo, el artículo 9 del Proyecto Cero introducía algunos nuevos elementos no recogidos en los Principios Rectores. Por ejemplo, se contemplaba que las empresas debían realizar consultas significativas con los grupos afectados, prestando una especial atención a grupos en situación de vulnerabilidad, como las mujeres, los niños y las niñas, las personas con discapacidad, los pueblos indígenas, las personas migrantes, refugiadas y desplazadas internas (artículo 9.2.g). A su vez, se preveía que las empresas debían contar con una garantía financiera para hacer frente a las posibles reclamaciones de indemnización (artículo 9.2.h). Finalmente, se proponía que incorporaran medidas de diligencia debida en todas las relaciones contractuales que suponen actividades empresariales de carácter transnacional (artículo 9.2.a).

La posición de los Estados y de las organizaciones de la sociedad civil respecto al artículo 9 del Proyecto Cero fue positiva. No obstante, la discusión en torno a esta disposición estuvo relacionada con la necesidad de una mayor precisión y alcance de los requisitos de

${ }^{4}$ La OCDE ha elaborado una serie de guías para ayudar a las empresas a llevar a cabo procesos de diligencia debida para alcanzar una conducta empresarial responsable en sectores específicos y cadenas de suministro: minerales, agricultura, textil y de calzado, industrias extractivas y finanzas. Véase http://www.oecd.org/corporate/mne/ 
diligencia debida en materia de derechos humanos, así como de una alineación con los conceptos y la terminología de los Principios Rectores o las guías sectoriales de la OCDE (CDH 2019). Esto, con el fin de no afectar consensos - materiales y formales - alcanzados por los diversos actores de interés sobre los estándares que las empresas deben cumplir para prevenir y rendir cuentas de sus impactos reales y potenciales sobre los derechos humanos.

Asimismo, surgieron dudas sobre la posibilidad que otorgaba el artículo 9 a los Estados de eximir a las pequeñas y medianas empresas de ciertas obligaciones contenidas en dicho artículo, ya que es contrario al espíritu del segundo pilar de los Principios Rectores que se aplica a todas las empresas atendiendo a sus capacidades (CDH 2019). En este sentido, los Principios Rectores proporcionan un enfoque más integrador que podría ser adoptado en el futuro instrumento, ya que reconocen que las pequeñas y medianas empresas pueden disponer de menor capacidad y, por tanto, sus respectivos procesos y políticas de diligencia debida adoptarán formas diferentes que sean proporcionales, entre otros factores, a su tamaño.

La disposición del artículo 9 del Proyecto Cero tampoco tomaba en consideración la complejidad de implementar la diligencia debida en zonas afectadas por conflictos. La vaguedad e imprecisión de los requisitos, según se encontraban en el Proyecto Cero, implicaba el riesgo de que los Estados adoptaran legislaciones divergentes y fragmentadas que puedan generar espacios en los que las empresas deslocalicen sus actividades para evitar el cumplimiento de requisitos más estrictos.

En la versión revisada del Proyecto la disposición de prevención es mucho más clara y está contemplada en el artículo 5. El nuevo texto se alinea mejor con lo dispuesto en los Principios Rectores, ya que impone a los Estados la obligación de asegurar en su legislación interna que todas las personas que realizan actividades empresariales, incluidas las de carácter transnacional, respeten los derechos humanos y prevengan las violaciones o abusos de derechos humanos. Para ello, se prevé que los Estados Partes adopten las medidas necesarias para la implementación de procesos de diligencia debida en todas las actividades empresariales.

Esta nueva disposición de la versión revisada del Proyecto incorpora de manera literal las etapas del proceso de diligencia debida en materia de derechos humanos contemplado en los Principios Rectores, al mismo tiempo que mantiene y mejora los nuevos elementos propuestos en el artículo 9 del Proyecto Cero. No obstante, el alcance previsto para estos procesos de diligencia debida se limita a las «actividades» de las 
empresas (business activities), incluidas las «relaciones contractuales» 5 (contractual relationships), en lugar de las «relaciones comerciales» (business relationships) como establecen los Principios Rectores. Esto crea el riesgo de que las empresas no aborden algunos de los impactos generados por entidades de la cadena de suministro que no cuenten con una relación contractual directa según las disposiciones del futuro instrumento.

El artículo 5 de la versión revisada del Proyecto reformula la posibilidad de eximir a las pequeñas y medias empresas y, en su lugar, contempla que los Estados pueden proporcionar incentivos y otras medidas para facilitar que estas empresas cumplan con las obligaciones de diligencia debida sin causar cargas adicionales que sean excesivas. Esta nueva disposición se aproxima más a lo establecido en los Principios Rectores y, sobre todo, se alinea con el enfoque del GTI de extender el ámbito de aplicación del tratado para todas las actividades empresariales, ya sean de carácter nacional o transnacional.

El potencial de la disposición de prevención del futuro tratado es que cristaliza la obligación de diligencia debida para las empresas. Se espera que los Estados introduzcan en su legislación interna la diligencia debida obligatoria en materia de derechos humanos. En este sentido, existen importantes desarrollos normativos en algunos Estados que se espera que se reproduzcan en otras jurisdicciones nacionales a partir del futuro instrumento.

En Francia, por ejemplo, se aprobó la Loi relative au devoir de viligance des sociétés mères et des entreprises donneuses d'ordre en 2017. En los Países Bajos, en mayo de 2019, el Senado votó a favor del Proyecto de Ley de diligencia debida en materia de trabajo infantil. Otros desarrollos similares son las leyes de Modern Slavery de 2018 en Australia y de 2015 en el Reino Unido, la California Transparency in Supply Act de 2010, la Directiva europea sobre divulgación de información no financiera y el Reglamento sobre los minerales de zonas de conflicto de la UE. En países como Suiza, Alemania, Australia, Bélgica y Finlandia, entre otros, se proponen iniciativas legislativas similares a las señaladas.

5 Según el artículo 1.4 de la versión revisada del Proyecto, la «relación contractual» se refiere a cualquier relación entre personas físicas o jurídicas para llevar a cabo actividades comerciales, incluidas, entre otras, aquellas actividades realizadas a través de filiales, subsidiarias, agentes, proveedores, cualquier asociación comercial, joint venture, empresa beneficiaria o cualquier estructura o relación contractual, según lo dispuesto en la legislación interna del Estado. 


\subsection{El derecho de las víctimas de acceso a la justicia y a la reparación efectiva}

Una de las aportaciones más significativas del futuro tratado son las disposiciones que reconocen los derechos de las víctimas ${ }^{6}$ de abusos corporativos y las de la responsabilidad jurídica de las empresas por violaciones o abusos de derechos humanos. Estas disposiciones están dirigidas a eliminar o reducir los obstáculos prácticos y legales que las víctimas enfrentan para acceder a los recursos judiciales y para hacer efectiva la responsabilidad de las empresas.

El artículo 8 propuesto en el Proyecto Cero del tratado se inspiró en la obligación de los Estados de garantizar a todas las personas el acceso justo, efectivo y rápido a la justicia y a los recursos efectivos ante los tribunales nacionales competentes. Por ello, esta disposición enlistó algunos de los siguientes derechos para las víctimas: i) acceso equitativo, efectivo y rápido a la justicia y a los recursos (restitución, compensación, rehabilitación, satisfacción y garantías de no repetición); ii) acceso adecuado a la información pertinente para la tramitación de los recursos; iii) asistencia jurídica adecuada y efectiva durante todo el proceso legal; iv) acceso a medios diplomáticos y consulares apropiados; iv) derecho a gozar de una consideración y atención especiales, para que los procedimientos destinados al acceso a la justicia y los recursos no den lugar a una nueva victimización.

Además de los derechos señalados anteriormente, llamaba la atención las disposiciones que intentaban nivelar la asimetría de recursos en los casos de empresas y derechos humanos. El artículo 8.5.d del Proyecto Cero señalaba que en ningún caso se requeriría a las víctimas que reembolsaran las costas judiciales de la otra parte de la reclamación. Si bien esta disposición contribuía a disminuir algunos de

6 Desde la perspectiva del derecho internacional, la versión revisada del Proyecto proporciona una definición novedosa y enriquecedora de lo que se entiende por «víctima». El artículo 1 señala que "víctima» es cualquier persona o grupo de personas que individual o colectivamente hayan sufrido o aleguen haber sufrido violaciones o abusos de los derechos humanos. En su caso, y de conformidad con la legislación nacional, el término «víctima» también incluye a la familia inmediata o dependientes de la persona directa. Por tanto, se trata de una de definición ampliada de la noción de "víctima» contenida en los Principios y directrices básicos sobre el derecho de las víctimas de violaciones manifiestas de las normas internacionales de derechos humanos y de violaciones graves del derecho internacional humanitario a interponer recursos y obtener reparaciones de 2005, ya que una simple alegación de haber sufrido daños será suficiente para que una persona o grupo de personas adquiera el estatus de «víctima». Sobre la definición de «víctima» en el tratado sobre empresas y derechos humanos, véase Angelini (2019). 
los posibles obstáculos financieros que enfrentan las víctimas, también podría generar el riesgo de crear una carga de trabajo excesiva a los tribunales nacionales por el posible aumento de casos frívolos y carentes de fundamento que no tienen oportunidad de éxito en sede judicial. En este sentido, el tratado debe establecer de manera clara los supuestos en los que se exime a las víctimas de las costas judiciales.

Asimismo, se ha contemplado la creación de un Fondo Internacional para las Víctimas cuyo funcionamiento se definirá una vez que entre en vigor el tratado. No obstante, las delegaciones han manifestado cierta reticencia por la posibilidad de que sean los mismos Estados quienes asuman la carga de resarcir los abusos cometidos por las empresas (CDH 2019).

Otras de las deficiencias que presentaba la disposición contemplada en el artículo 8 del Proyecto Cero es la falta de garantías especiales para abordar la situación de las personas defensoras de los derechos humanos. Tampoco se tuvo en cuenta a los colectivos o grupos que se ven especialmente afectados por las violaciones de derechos humanos cometidas por empresas o que tienen mayores dificultades para lograr un acceso efectivo a la justicia. En este sentido, se sugirió que esta disposición del futuro instrumento integre una perspectiva de género y una referencia expresa a la protección de las personas defensoras de derechos humanos (CDH 2019).

La versión revisada del Proyecto atiende en su gran mayoría las inquietudes surgidas durante el cuarto período de sesiones del GTI respecto a la disposición de los derechos de las víctimas. El artículo 5 de la versión revisada mantiene la disposición que estipula que en ningún caso se requerirá a las víctimas el reembolso de las costas judiciales de la otra parte de la reclamación, pero agrega que esto se hará efectivo siempre y cuando la presunta víctima demuestre que dicho reembolso no puede realizarse debido a la falta o insuficiencia de recursos económicos. Asimismo, uno de los avances más relevantes es que se incluye una nueva disposición que señala que los Estados Partes tomarán medidas adecuadas y efectivas para garantizar un entorno seguro y propicio para las personas, grupos y organizaciones que promueven y defienden los derechos humanos y el medio ambiente, de modo que puedan actuar sin amenazas, restricciones e inseguridad.

Por su parte, el artículo 10 del Proyecto Cero abordaba la responsabilidad jurídica de las empresas en caso de abusos de derechos humanos cometidos en el marco de sus actividades empresariales de carácter transnacional. Esta disposición adoptaba una posición flexible para dar a los Estados la libertad a la hora de determinar cuál era la mejor forma de aplicar el artículo, señalando que los Estados Partes 
deberían garantizar, por medio de su derecho interno, que se exigieran responsabilidades de índole penal, civil o administrativa a las personas físicas y jurídicas por violaciones de derechos humanos cometidas en el contexto de las actividades empresariales de carácter transnacional.

En materia de responsabilidad civil, el Proyecto Cero contenía una disposición que intentaba dar una solución a la responsabilidad jurídica de la empresa matriz por los abusos cometidos por sus filiales o entidades en la cadena de suministro. El levantamiento del velo corporativo es una de las cuestiones a la que los Principios Rectores no han podido brindar orientaciones concretas (De Schutter 2016). El artículo 10.6 del Proyecto Cero indicaba los supuestos en los que una empresa matriz podía ser considerada responsable por los daños de las consecuencias negativas sobre los derechos humanos de las operaciones que controla o de las que tenga una relación suficientemente estrecha con la filial o entidad en su cadena de suministro. Asimismo, podía incurrir en responsabilidad cuando hayan previsto o se debieran haber previsto riesgos de abusos de derechos humanos en su cadena de actividad económica.

A pesar del avance que representaba esta disposición, requería una mayor claridad y precisión, ya que introducía términos usados por primera vez en todo el Proyecto Cero como «control»o «relación suficientemente estrecha», sin proporcionar una definición. La falta de una delimitación de estos conceptos puede permitir la elusión de responsabilidades por parte de las empresas matrices respecto a los abusos cometidos por sus filiales (Guamán 2018). Sobre todo, puede llegar a resultar un incentivo perverso que evite que las matrices influyan en las actividades de sus filiales por el riesgo legal de incurrir en una responsabilidad en caso de abusos de derechos humanos (Fasciglione 2018). Esto resulta contradictorio a los Principios Rectores, que indican que las empresas deben ejercer su capacidad de influencia para prevenir o mitigar consecuencias negativas sobre los derechos humanos de las operaciones, productos o servicios prestados por otra entidad con la que mantienen relaciones comerciales (Principio 19).

Otra de las cuestiones más debatibles de esta disposición fue la responsabilidad penal contemplada en el artículo 10.8 del Proyecto Cero, que estipulaba que los Estados Parte dispondrán medidas para establecer la responsabilidad penal de todas las personas con actividades empresariales. En principio, la disposición de este artículo parecía imponer la obligación a los Estados de adoptar medidas para establecer la responsabilidad penal de todas las personas con actividades empresariales de carácter transnacional, a pesar de que aún hay Estados que no reconocen la responsabilidad penal de las 
personas jurídicas en sus ordenamientos jurídicos internos (Iglesias Márquez 2018a). No obstante, el artículo 10.12 introducía una vía de escape, ya que indicaba que en caso de que, según el ordenamiento jurídico de un Estado Parte, la responsabilidad penal no sea aplicable a las personas jurídicas, ese Estado velará por que estas estén sujetas a sanciones eficaces, proporcionadas y disuasorias de carácter no penal.

El artículo 10 del Proyecto Cero no contemplaba disposiciones específicas que orienten a los Estados a exigir la responsabilidad administrativa de las empresas por abusos de derechos humanos, sin mencionar la falta de consideración a los mecanismos no judiciales. En este sentido, se obviaba el potencial de algunas sanciones administrativas, como podía ser la exclusión de las empresas de participar en procedimientos de contratación pública, acceder a subvenciones estatales o créditos a la exportación y obtener garantías públicas para inversiones o créditos a la exportación.

Las disposiciones del artículo 10 del Proyecto Cero generaron bastantes discrepancias entre los Estados. Por ello, el artículo sobre la responsabilidad jurídica es uno de los que a primera vista presenta más cambios en la versión revisada del Proyecto (artículo 6). Aunque se sigue manteniendo un enfoque flexible y orientaciones específicas para los regímenes de responsabilidad civil y penal, el nuevo artículo 6 refleja los avances que han surgido en el derecho internacional y en la práctica nacional respecto a la responsabilidad de las empresas por abusos de derechos humanos (López 2019).

Los avances más significativos del artículo 6 de la versión revisada del Proyecto son, por un lado, una mayor relación con las disposiciones de prevención, ya que presenta un lenguaje más concreto y preciso en relación con la responsabilidad de las empresas que controlan o supervisan por no prevenir que otra persona física o jurídica con la que tenga una relación contractual cause daños a terceros, que debería prever o haber previsto en el desarrollo de las actividades empresariales, incluidas las de carácter transnacional e independientemente de dónde se lleve a cabo la actividad. En este sentido, se intenta avanzar en la propuesta de un "sistema integral y adecuado» para hacer efectiva la responsabilidad de las empresas, no solo por los abusos de derechos humanos causados en el marco de sus actividades, sino también por su acción u omisión en aquellos cometidos por otras entidades en la cadena de suministro, sin importar el lugar donde ocurren, pero siempre y cuando exista una relación contractual directa.

Por otro lado, el artículo 6 contiene una lista de once delitos reconocidos por el derecho internacional, incluidos los crímenes del Estatuto de Roma, que darán lugar a la responsabilidad — penal, civil o 
administrativa - de las empresas por actos que constituyen un intento, participación o complicidad en los ilícitos contemplados para el futuro instrumento. Esta lista de delitos fortalece el texto del tratado, ya que lo dota de una mayor seguridad jurídica. A su vez, contribuye a que los Estados que ya reconocen la responsabilidad penal de las empresas incluyan nuevos tipos penales a sus catálogos de delitos atribuibles a estas entidades. A pesar del gran avance que representa esta nueva disposición del artículo 6, aún se mantiene la potestad discrecional de los Estados de elegir el tipo de responsabilidad según los principios de su derecho interno. Este enfoque potestativo reduce la posibilidad de que los Estados manifiesten oposiciones o reservas a esta disposición.

\subsection{La asistencia y cooperación internacional para implementación del tratado}

Uno de los factores que contribuye al contexto de impunidad en el que operan las empresas es la falta de cooperación entre los Estados para asegurar recursos efectivos de reparación para las víctimas de abusos corporativos (De Schutter 2016). Por ello, el GTI ha contemplado para el futuro tratado sobre empresas y derechos humanos la inclusión de disposiciones relacionadas con la asistencia judicial recíproca y la cooperación internacional, con el fin de garantizar la implementación efectiva del instrumento y para fortalecer los esfuerzos de los Estados de respetar y proteger los derechos humanos en el marco de las prácticas económicas contemporáneas. En virtud del futuro instrumento se espera que los Estados Parte cooperen de buena fe para iniciar y llevar a cabo investigaciones, procesos y actuaciones judiciales relacionadas con casos transfronterizos de abusos de derechos humanos cometidos por empresas.

Para asegurar la asistencia judicial recíproca, los Estados Parte deben considerar la posibilidad de celebrar, o en su defecto enmendar, acuerdos o arreglos bilaterales o multilaterales, así como la creación de órganos mixtos de investigación. No obstante, los Estados han manifestado algunas preocupaciones en relación con esta disposición, principalmente por la falta de consideración de las posibles dificultades que algunos países pueden tener para implementarla y al desequilibrio respecto a los derechos procesales de los acusados. Asimismo, alguna delegación propuso incorporar un componente de doble tipicidad para evitar el uso indebido de la asistencia judicial recíproca (CDH 2019).

En relación con la cooperación internacional, se espera que se adopten medidas entre los Estados Parte, las organizaciones 
internacionales y regionales pertinentes y la sociedad civil con el fin de: fomentar la cooperación técnica eficaz y la creación de capacidades; compartir experiencias, buenas prácticas, dificultades, información y programas de capacitación; y facilitar la cooperación en la investigación y estudios sobre las mejores prácticas y experiencias para prevenir las violaciones de los derechos humanos. Tanto los Estados como las organizaciones de la sociedad civil sugieren que esta disposición debería hacer referencia a temas transfronterizos de manera más explícita y permitir a las empresas unirse formalmente a los esfuerzos de cooperación internacional (CDH 2019).

Las disposiciones de asistencia judicial y cooperación internacional no son desconocidas para los Estados, ya que están contenidas en diversos tratados de derechos humanos. Estas disposiciones del futuro instrumento contribuyen a que los mecanismos de reparación existentes a nivel nacional funcionen más eficazmente, superando así algunos de los obstáculos que pueden resultar de las actividades transnacionales de las empresas (De Schutter 2016). Se trata, por tanto, de la implementación del tercer pilar de los Principios Rectores a través de la cooperación y asistencia judicial internacional. Asimismo, sirve para disminuir las tensiones entre los Estados que se pueden generar del ejercicio de la jurisdicción extraterritorial (Deva 2018b).

Por lo anterior, los artículos 11 (asistencia judicial recíproca) y 12 (cooperación internacional) del Proyecto Cero no generaron gran controversia. En consecuencia, el contenido de estas disposiciones no supuso grandes cambios en la versión revisada del Proyecto. Quizás el único cambio que se identifica es que el requerimiento a los Estados Partes de cooperar de buena fe se ubica ahora en el párrafo inicial del nuevo artículo 11 sobre cooperación internacional, en lugar del párrafo inicial del artículo sobre asistencia judicial recíproca, donde estaba anteriormente en el Proyecto Cero. Este cambio da una mayor claridad y coherencia al texto.

\subsection{Los mecanismos institucionales de supervisión y cumplimiento}

El GTI ha propuesto la creación de un Comité de expertos independientes para supervisar el cumplimiento de las disposiciones del futuro instrumento, dejando así a un lado la propuesta de establecer un tribunal o un centro de supervisión internacional en materia de empresas y derechos humanos, como proponen algunas organizaciones de la sociedad civil y académicos (Hernández Zubizarreta 2016; Bilchitz 2016). 
Las funciones propuestas para el Comité son las siguientes: formular observaciones generales, examinar y brindar las observaciones y recomendaciones finales que estime oportunas con respecto a los informes presentados por los Estados Parte, apoyar a los Estados Parte en la tarea de recopilar y transmitir la información necesaria para garantizar la aplicación del tratado, y presentar un informe anual sobre sus actividades a los Estados Parte y a la Asamblea General de las Naciones Unidas, entre otras. ${ }^{7}$ Por su parte, el Proyecto del Protocolo Facultativo reconoce la competencia del Comité de recibir comunicaciones de individuos o grupos relacionadas con violaciones de derechos humanos cometidas en la jurisdicción de un Estado Parte del Protocolo. Esta función del Comité permite no solo detener una violación en curso, sino también establecer algunas orientaciones para que los Estados prevengan violaciones de derechos humanos en situaciones similares (Deva 2018b).

Las competencias previstas se alejan de la propuesta de supervisar las actividades de millones de empresas operando a nivel global; una tarea que, en términos prácticos, es imposible de llevar a cabo por un Comité con recursos limitados. Sus funciones se asemejan a la de otros órganos de los tratados y, por ende, se centran principalmente en la promoción, interpretación y aplicación del futuro tratado a la luz de las obligaciones internacionales de los Estados.

A pesar de que la creación de un Comité puede ser un aspecto positivo para asegurar la implementación del futuro instrumento, no se debe obviar la experiencia de otros órganos similares ni las diversas recomendaciones formuladas por expertos y académicos para mejorar el sistema de los órganos creados en virtud de los tratados de derechos humanos, incluidas aquellas sobre el fortalecimiento de la independencia y la experiencia de los miembros de los órganos de los tratados, mayor accesibilidad para la presentación de comunicaciones individuales, simplificación del proceso de presentación de informes y el seguimiento efectivo de las observaciones finales de los Comités, entre otras (Geneva Academy 2018).

En algunos casos se ha identificado que los miembros que conforman los Comités ocupan, a su vez, cargos políticos en sus Estados, lo que afecta a la independencia de los órganos (Geneva Academy 2018). En este sentido, la versión revisada del Proyecto incluye una especificación expresa de que los Estados Parte

7 Véase artículo 14 del Proyecto Cero y artículo 13 de la versión revisada del Proyecto. 
deberán asegurar que los expertos elegidos no participen, directa o indirectamente, en ninguna actividad que pueda afectar adversamente el propósito de tratado. En otros casos, la calidad de la experiencia de los miembros es desigual debido a un proceso de selección de carácter altamente político en el que los Estados están dispuestos a negociar la designación de los miembros de los órganos (Crawford 2000). En el ámbito de empresas y derechos humanos, los miembros del Comité deben ser capaces de abordar cuestiones multidisciplinarias de manera despolitizada en sus observaciones generales e informes, sobre todo teniendo en cuenta la complejidad y transversalidad de esta materia.

Finalmente, en relación con este mecanismo de supervisión, surgen dudas sobre el financiamiento de este órgano, ya que no se han previsto hasta el momento disposiciones sobre esta cuestión específica (CDH 2019). La asignación de los recursos financieros a los órganos de los tratados depende en gran medida de la voluntad política de los Estados, que en muchas ocasiones no han aprobado el aumento de presupuesto para que desarrollen sus funciones de manera más eficiente. La falta de fondos genera una situación de vulnerabilidad para dichos órganos, lo que dificulta a su vez que puedan cumplir con sus mandatos y con las crecientes cargas de trabajo que suelen tener (Schimdt 2000).

Por otra parte, se contempla la organización de una Conferencia de las Partes (COP) para abordar cuestiones relacionadas con la aplicación del futuro instrumento. La COP abriría la puerta a más negociaciones para el desarrollo de nuevos avances y mecanismos en el ámbito de empresas y derechos humanos que puedan ser adoptados en ulteriores protocolos. Esto permitiría que el instrumento se adaptara a futuros cambios en la coyuntura política y social de la comunidad internacional. Finalmente, el Proyecto de Protocolo Facultativo contempla también un mecanismo nacional de implementación que los Estados Parte deben crear en su jurisdicción, con el fin de asegurar el cumplimento de las disposiciones del tratado y un mecanismo de reparación para las personas afectadas por las actividades empresariales.

\section{Conclusiones}

En los últimos años la dinámica de la agenda internacional -y de algunos Estados - en materia de empresas y derechos humanos ha presentado desarrollos considerables que eran impensables hace cuarenta años. La adopción de un tratado en la materia es, sin lugar a dudas, uno de los siguientes pasos que ha estado pendiente en la 
agenda internacional, sobre todo teniendo en cuenta la actual crisis de derechos humanos y ambiental en la que estamos inmersos. Un instrumento de este tipo reconfigura y actualiza la arquitectura del derecho internacional de los derechos humanos para hacer frente a los retos y a la nueva realidad generada por el avanzado proceso de globalización económica. Asimismo, podría representar un cambio tajante a la posición predominante de que únicamente los Estados tienen las obligaciones de proteger los derechos humanos, y que únicamente los actores estatales pueden cometer violaciones de derechos humanos reconocidos internacionalmente.

El proceso de elaboración del tratado en el seno de las Naciones Unidas no solo ha servido para demostrar y denunciar el contexto geopolítico y geoeconómico en el que operan actualmente las empresas, sino también para alzar las voces - como nunca antesde las víctimas que sufren las peores externalidades de las actividades empresariales a nivel global. Los períodos de sesiones del GTI han convocado la participación histórica de un gran número de Estados, empresas y de diversos grupos de la sociedad civil de todo el mundo. El Sur Global concibe este proceso como una oportunidad única de adoptar un instrumento que articule acciones colectivas para nivelar las asimetrías de poder, cubrir los vacíos de gobernanza, acabar con la impunidad corporativa y atender las diferentes necesidades generadas en el marco de la dicotomía Norte-Sur. Por tanto, se trata de un proceso que ha demostrado avances significativos en las negociaciones y en el que se debe asegurar la participación de diversos actores de varias regiones del mundo para la legitimación y construcción de un instrumento internacional con un enfoque bottom-up.

El futuro tratado es, por tanto, una posible contribución importante a la protección de los derechos humanos con el potencial de cambiar las dinámicas de los principales actores de la economía mundocapitalista. No obstante, no debe concebirse como una solución holística para abordar todas las cuestiones planteadas en el ámbito de empresas y derechos humanos, sino más bien como un instrumento normativo adicional en el complejo universo de iniciativas que tienen como objetivo asegurar la responsabilidad de las empresas de respetar y promover los derechos humanos en el marco de sus actividades. Por tanto, se trata de un instrumento que refuerza las obligaciones y compromisos de los Estados. De hecho, en virtud del derecho internacional contemporáneo, los Estados ya tienen la obligación de proteger los derechos humanos frente a las actividades particulares, incluidas las de las empresas. El tratado propuesto en su estado actual es simplemente un catalizador que unifica y propone principios y 
mecanismos internacionales que fortalecerían la capacidad de los Estados para cumplir con esta obligación cuando las violaciones de derechos humanos son cometidas en el desarrollo de actividades empresariales de carácter transnacional.

El posible futuro tratado no ha logrado incluir disposiciones que ponen a prueba las fronteras del derecho internacional, sino más bien se ha limitado a aquellos puntos en los que los diversos actores han llegado a un consenso político. Por ello, el instrumento propuesto adopta la visión clásica del derecho internacional. Si bien este enfoque facilita su eventual adopción, a su vez, requiere de un compromiso real de los Estados para desdoblar e implementar su contenido dentro de su jurisdicción. Por tanto, es necesario seguir trabajando en las incoherencias y ambigüedades que presentan las disposiciones del futuro instrumento para seguir desarrollando y clarificando aquellos elementos que presentan importantes potencialidades para contrarrestar la impunidad corporativa y asegurar la efectiva reparación a las víctimas. En consecuencia, el futuro tratado es solo un paso más en la correcta dirección para asegurar que las empresas respeten los derechos humanos en el marco de sus actividades globales y, a su vez, es un instrumento que debe coexistir y complementar otras iniciativas -voluntarias y obligatorias - que comparten el objetivo común de promover una conducta empresarial responsable.

\section{Bibliografía}

Angelini, Antonella. 2019. "What the draft treaty's definition of 'victim' means for access to remedy". Acceso el 19/09/2019. https://www.businesshumanrights.org/en/what-the-draft-treatys-definition-of-victim-means-foraccess-to-remedy

Anghie, Antony. 2006. "The Evolution of International Law: Colonial and Postcolonial Realities". Third World Quarterly 27 (5): 739-753.

Bilchitz, David. 2016. "The Necessity for a Business and Human Rights Treaty". Business and Human Rights Journal 1 (2): 203-227.

Brown, Julia G. 2013. "International Investment Agreements: Regulatory Chill in the Face of Litigious Heat?" Western Journal of Legal Studies 3 (1): $1-27$.

Cantú Rivera, Humberto. 2018. "Some remarks on the third sessions of the Business and Human Rights Treaty Process and the «Zero Draft»". Brazilian Journal of International Law 15 (2): 25-40.

Cassel, Douglass. 2018. "The Third Session of the UN Intergovernmental Working Group on a Business and Human Rights Treaty". Business and Human Rights Journal 3 (2): 277-283. 
Cassel, Douglass. 2018a. "Opciones para un tratado sobre empresas y derechos humanos. Avances y estado del arte". En: El constitucionalismo transformador en América Latina y el derecho económico internacional. De la tensión al diálogo, coordinado por Armin von Bogdandy et al., 211245. Ciudad de México: UNAM-IIJ.

Cassel, Douglass. 2019. "Ecuador's Revised Draft Treaty: Getting Down to Business". Acceso el 21/09/2019. https://www.cambridge.org/core/ blog/2019/09/09/ecuadors-revised-draft-treaty-getting-down-to-business/

CDH. 2016. "Informe del primer período de sesiones del grupo de trabajo intergubernamental de composición abierta sobre las empresas transnacionales y otras empresas con respecto a los derechos humanos, con el mandato de elaborar un instrumento internacional jurídicamente vinculante". Acceso el 21/05/2019. http://ap.ohchr.org/documents/ dpage_e.aspx?si=A/HRC/31/50.

$\mathrm{CDH}$. 2018. "Informe del tercer período de sesiones del grupo de trabajo intergubernamental de composición abierta sobre las empresas transnacionales y otras empresas con respecto a los derechos humanos". Acceso el 09/06/2019. http://ap.ohchr.org/documents/dpage_e.aspx?si=A/ $\mathrm{HRC} / 37 / 67$.

CDH. 2019. "Informe del Grupo de Trabajo intergubernamental de composición abierta sobre las empresas transnacionales y otras empresas con respecto a los derechos humanos sobre su cuarto período de sesiones". Acceso el 21/05/2019. http://ap.ohchr.org/documents/dpage_e. aspx?si=A/HRC/40/48.

Čeernič, Jernej Letnar y Carrillo Santarelli, Nicolás. 2018. The future of business and Human Rights. Theoretical and practical considerations for a UN Treaty. Cambridge: Intersentia.

Crawford, James 2000. "The UN human rights treaty system: A system in crisis?" En: The Future of UN Human Rights Treaty Monitoring, editado por Philip Alston y James Crawford, 1-12. Cambridge: Cambridge University Press.

De Schutter, Olivier. 2016. "Towards a New Treaty on Business and Human Rights". Business and Human Rights Journal 1 (2): 41-67.

Delegación de Bolivia. 2017. "Estado Plurinacional de Bolivia, Panel VI sobre las obligaciones de Empresas Transnacionales y otras empresas". Acceso el 21/05/2019. www.ohchr.org/Documents/HRBodies/HRCouncil/ WGTransCorp/Session1/Panel6/States/Bolivia_Plurinational_State_of.pdf

Delegación de Ecuador. 2017. "Mejorando la responsabilidad de las empresas transnacionales y otras empresas de negocios con respecto a los derechos humanos, incluyendo prevención, mitigación y remediación". Acceso el 21/05/2019. www.ohchr.org/Documents/HRBodies/HRCouncil/ WGTransCorp/Session1/Panel6/States/Ecuador.pdf

Delegación de la Unión Europea. 2017. "Opening remarks by the European Union, working group on transnational corporations and other business enterprises with respect to human rights 3rd session (23-27 October 2017)". Acceso el 21/05/2019. www.ohchr.org/Documents/HRBodies/ 
HRCouncil/WGTransCorp/Session3/Orallnterventions/EUOpening_remarks. pdf

Delegación de los Estados Unidos. 2014. "Proposed Working Group Would Undermine Efforts to Implement Guiding Principles on Business and Human Rights". Acceso el 22/05/2019. https://geneva.usmission. gov/2014/06/26/proposed-working-group-would-undermine-efforts-toimplement-guiding-principles-on-business-and-human-rights/

Deva, Surya y Bilchitz, David. 2013. Human Rights Obligations of Business: Beyond the Corporate Responsibility to Respect. Cambridge: Cambridge University Press.

Deva, Surya. 2018a. "Scope of the Proposed Business and Human Rights Treaty Navigating through Normativity, Law and Politics". En: Building a Treaty on Business and Human Rights: Context and Contours, editado por Surya Deva y David Bilchitz, 154-182. Cambridge: CUP.

Deva, Surya. 2018b. "The Zero Draft of the Proposed Business and Human Rights Treaty, Part II: On the Right Track, but Not Ready Yet". Acceso el 22/05/2019. https://www.business-humanrights.org/en/the-zero-draft-ofthe-proposed-business-and-human-rights-treaty-part-ii-on-the-right-trackbut-not-ready-yet

Fasciglione, Marco. 2018. "Another Step on the Road? Remarks on the Zero Draft Treaty on Business and Human Rights". Diritti Umani e Diritto Internazionale 12 (3): 629-661.

Geneva Academy. 2018. Optimizing the UN Treaty Body System. Ginebra: The Geneva Academy of International Humanitarian Law and Human Rights.

Gonzalez, Carmen. 2015. "Environmental Justice, Human Rights, and the Global South". Santa Clara Journal of International Law 13: 151-195.

Gordon, Ruth y Sylvester, Jon H. 2011. "Deconstructing Development". Wisconsin International Law Journal, 22: 1-98.

Guamán, Adoración. 2018 "El Draft 0 del Binding Treaty: análisis crítico del contenido del texto y su adecuación con el objetivo de la Resolución 26/9". Cadernos de Pesquisa Homa 1 (6): 1-35.

Hernández Zubizarreta, Juan. 2009. Las empresas transnacionales frente a los derechos humanos: Historia de una asimetría normativa. De la responsabilidad social corporativa a las redes contra hegemónicas transnacionales. Bilbao-Vitoria/Gasteiz: Hegoa-OMAL.

Hernández Zubizarreta, Juan. 2016. “El Tribunal Internacional para las ETN y los DDHH". America Latina en movimiento, 520: 14-15.

Iglesias, Daniel. 2018a. "La responsabilidad penal de las empresas por graves violaciones de derechos humanos: práctica actual y desafíos futuros". Brazilian Journal of International Law 15 (2): 130-149.

Iglesias, Daniel. 2018b. "Explorando las fronteras del Derecho internacional de los derechos humanos: hacia la adopción de un tratado internacional sobre empresas y derechos humanos". Akademía. Revista Internacional y Comparada de Derechos Humanos 1 (1): 165-214.

IOE, ICC, BIAC, WBCSD. 2016. "UN Treaty Process on Business and Human Rights. Further considerations by the international business community 
on a way forward Second Submission". Acceso el 21/05/2019. https:// iccwbo.org/publication/icc-ioe-biac-wbcsd-un-treaty-business-humanrights-futher-considerations/

León, M. ${ }^{a}$ Augusta y Liu, Bingyu. 2018. "The Challenge of Bringing Transnational Corporations to Justice for Environmental Damage: A Case Study of Texaco Oil Company and Chevron vs Lago Agrio Plaintiffs". En: Courts and the Environment, editado por Christina Voigt y Zen Makuch, 271-289. Cheltenham-Northampton: Edward Elgar.

López, Carlos. 2019. "Legal liability for business human rights abuses under the revised draft of a treaty on business and human rights". Acceso el 19/09/2019. https://www.cambridge.org/core/blog/2019/09/11/legalliability-for-business-human-rights-abuses-under-the-revised-draft-of-atreaty-on-business-and-human-rights/

Miles, Kate. 2010. "International Investment Law: Origins, Imperialism and Conceptualizing the Environment". Colorado Journal of International Environmental Law and Policy 21 (1): 1-47.

República del Ecuador. 2013. "Statement on Behalf of a Group of Countries at the 24rd Session of the Human Rights Council". Acceso el 21/05/2019. https://www.business-humanrights.org/sites/default/files/media/ documents/statement-unhrc-legally-binding.pdf

Ruggie, John. 2014. ¿Solamente negocios? Multinacionales y derechos humanos. Barcelona: Icaria.

Sauvant, Karl. 2015. "The Negotiations of the United Nations Code of Conduct on Transnational Corporations. Experience and Lessons Learned". Journal of World Investment \& Trade 16 (1): 11-87.

Schmidt, Markus. 2000. "Servicing and financing human rights supervision". En: The Future of UN Human Rights Treaty Monitoring, editado por Philip Alston y James Crawford, 481-498. Cambridge: Cambridge University Press.

Simons, Penelope. 2012. "International Law's Invisible Hand and the Future of Corporate Accountability for Violations of Human Rights". Journal of Human Rights and the Environment 3 (1): 5-43.

Vázquez, Carlos M. 2005. "Direct vs. Indirect Obligations of Corporations under International Law". Columbia Journal of Transnational Law 43 (3): 927-955.

Weissbrodt, David y Kruger, Muria. 2003. "Norms on the Responsibilities of Transnational Corporations and Other Business Enterprises with Regard to Human Rights". American Journal of International Law 97 (4): 901-922. 


\section{Copyright}

Deusto Journal of Human Rights / Revista Deusto de Derechos Humanos is an Open Access journal; which means that it is free for full and immediate access, reading, search, download, distribution, and reuse in any medium only for non-commercial purposes and in accordance with any applicable copyright legislation, without prior permission from the copyright holder (University of Deusto) or the author; provided the original work and publication source are properly cited (Issue number, year, pages and DOI if applicable) and any changes to the original are clearly indicated. Any other use of its content in any medium or format, now known or developed in the future, requires prior written permission of the copyright holder.

\section{Derechos de autoría}

Deusto Journal of Human Rights / Revista Deusto de Derechos Humanos es una revista de Acceso Abierto; lo que significa que es de libre acceso en su integridad inmediatamente después de la publicación de cada número. Se permite su lectura, la búsqueda, descarga, distribución y reutilización en cualquier tipo de soporte sólo para fines no comerciales y según lo previsto por la ley; sin la previa autorización de la Editorial (Universidad de Deusto) o la persona autora, siempre que la obra original sea debidamente citada (número, año, páginas y DOI si procede) y cualquier cambio en el original esté claramente indicado. Cualquier otro uso de su contenido en cualquier medio o formato, ahora conocido o desarrollado en el futuro, requiere el permiso previo por escrito de la persona titular de los derechos de autoría. 